ISSN 1997-5902

\title{
Action du fongicide naturel NECO contre la cercosporiose noire (Mycosphaerella fijiensis Morelet) chez le bananier plantain (AAB) en Côte d'Ivoire
}

\author{
KASSI Fernand Martial ${ }^{1 *}$, BADOU Odjoutchoni Jean ${ }^{1}$, TONZIBO Z. Félix², SALAH Ziane ${ }^{1}$ AMARI Ler- \\ N'ogn Dadé Georges Elisée', KONE Daouda1 \\ 1- Laboratoire de physiologie végétale, UFR Biosciences, Université Félix Houphouët Boigny, 22 BP 582 Abidjan 22, \\ Côte-d'Ivoire \\ 2. Laboratoire de chimie organique biologique, UFR SSMT, Université Félix Houphouët Boigny, 22 BP 582 Abidjan \\ 22, Côte-d'Ivoire \\ *Email: fernand2kassi@yahoo.fr \\ Original submitted in on $1^{\text {st }}$ November 2013. Published online at www.m.elewa.org on $31^{\text {st }}$ March 2014. \\ http://dx.doi.org/10.4314/jab.v75i1.3
}

\section{RESUME}

Objectifs : La présente étude a été conduite dans le but de déterminer l'effet du biofongicide codifié NECO sur la sévérité de Mycosphaerella fijiensis, champignon responsable de la maladie des raies noires (MRN) sur deux cultivars de bananier plantain (Corne 1 et Orishele) en condition d'infestation naturelle.

Méthodologie et résultats : Le NECO, obtenu par entrainement à la vapeur d'eau des feuilles fraiches de Ocimum gratissimum dans un dispositif de type Clevenger a été pulvérisé à raison de $5 \mathrm{ml} / \mathrm{l}$ en mélange avec l'huile minérale banole. Les applications du NECO sur les feuilles de bananiers dans les parcelles traitées sont faites au seuil de 2000 unités correspondant à la valeur de l'état d'évolution de la maladie (EEM) des raies noires sur 10 bananiers traités. 4 applications du NECO ont été réalisées au cours de la période d'évaluation (Novembre 2010- Avril 2011). Sur les parcelles traitées avec le NECO, l'évaluation hebdomadaire de quelques descripteurs phytopathologiques (PJFT, PJFN, PJF3, EEM, IS) de la MRN a révélé des niveaux plus faibles d'infection par la cercosporiose noire. Les valeurs de l'EEM ont été significativement réduites après chaque traitement, le rang de la plus jeune de feuille touchée (PJFT) est demeuré supérieur à celui évalué sur les parcelles témoins, le rang de la plus jeune feuille nécrosée (PJFN) a été supérieur à 6 et l'indice de sévérité de la maladie (IS) s'est annulée à la $16^{\mathrm{e}}$ semaine d'observation.

Conclusion et application de résultats : La pulvérisation foliaire avec le NECO peut constituer un moyen efficace de lutte biologique contre Mycosphaerella fijiensis et peut être associé dans un système de lutte intégré contre la maladie des raies noires en bananeraie.

Mots clés : Bananiers plantains, Mycosphaerella fijiensis, NECO, Lutte biologique, Côte d'Ivoire

\section{ABSTRACT}

Objectives: The present study was to determinate effect of a natural fungicide coded NECO on Mycosphaerella fijiensis severity, fungi responsible of the black Sigatoka disease (BSD) on two cultivars of plantain (Corne 1 and Orishele) in natural infection condition. 
Methodology and Results: NECO was obtained by extraction of essential oil from fresh leaves of Ocimum gratissimum and sprayed sprayed in a mixture with banole oil at $5 \mathrm{ml} / \mathrm{l}$. The application of NECO on banana leaves was done at 2000 units corresponding of disease state development (DSD)of 10 plantain trees treated. 4 applications of NECO were done during the period of evaluation (November 2010 - April 2011). Weekly evaluation of some phytopathological descriptors (PJFT, PJFN, PJF3, EEM, IS) of BSD on field treated revealed lower levels of infection by the black Sigatoka. Values of the EEM (DSD) were significantly reduced after each treatment, youngest leave touched by the disease (PJFT)is remained higher than those of untreated field, level of youngest leave necrosed (PJFN) was higher than 6 and index of disease severity (IS) was cancelled at the 16 th week of observation.

Conclusion and application of findings: Foliar spray spray with the NECO can be used as biological mean of fight against Mycosphaerella fijiensis and can be associated in an integrated system to to fight against black Sigatoka disease in banana field.

Keywords: Plantain, Mycosphaerella fijiensis, NECO, Biological Control Control, Côte d'Ivoire

\section{INTRODUCTION}

La cercosporiose noire ou maladie des raies noires (MRN), causée par le champignon ascomycète Mycosphaerella fijiensis MORELET (Stover, 1980) est la maladie foliaire la plus préjudiciable à la production de bananes à travers le monde (Pasberg-Gaulh et al., 2000). En Côte d'Ivoire, cette maladie détectée en 1985 dans la région d'Aboisso (Mourichon \& Fullerton, 1990), est aujourd'hui présente dans toutes les zones de culture du bananier notamment dans la région des 18 montagnes où elle cohabite avec la cercosporiose jaune (Camara, 2011). Les dégâts causés par cette maladie se traduisent par des pertes de rendement pouvant atteindre $100 \%$ et provoquent la maturité précoce des bananes (Ramsey et al, 1990 ; Chillet et al., 2009). Afin de lutter efficacement contre le parasite, les fongicides de synthèse de la famille des benzimidazoles, des triazoles et des strobulurines sont essentiellement utilisés mais entraînent des coûts de production élevés, l'induction de résistance de la part des agents pathogènes (Essis, 2010) et une contamination des bananes et de l'environnement (Rodríguez \& Jiménez 1985, Fullerton \& Olsen 1991, Mouliom Pefoura 1999). Des solutions alternatives en utilisant des produits d'origine naturels non polluants, ont des avantages certains sur les produits chimiques car ils sont moins nocifs pour l'écosystème et parce que la microflore environnementale elle-même biodégrade in situ pour les transformer en composés non toxique (Sánchez Rodriguez et al., 2002). Pour une agriculture durable, la recherche de solutions alternatives à l'emploi de pesticides de synthèse mais utilisant des composés naturels ne polluant pas l'environnement s'avère être important. L'huile essentielle extraite des feuilles de Ocimum gratissimum (basilic sauvage), une plante très répandue et utilisée dans la pharmacopée ivoirienne (Adjanohoun, 1979) est soupçonnée avoir des propriétés antifongiques contre les bioagresseurs. La composition chimique de cette huile est variable et 5 différents chémotypes ont été décrits, caractérisés par le constituant majoritaire de l'huile (eugénol, thymol, citral, ethyl cinnamate et linalool) Kishore Dubey et al., (2000). En Côte d'Ivoire, l'analyse chimique a permis d'identifier le chémotype à Thymol/paracymène (Oussou et al., 2004). En outre, les études réalisées par Camara et al (2007) ont montré que I'huile de cette plante avait un effet antifongique sur Deightoniella torulosa (SYD.) ELLIS, parasite foliaire des bananiers. Dans les expérimentations réalisées au champ, l'action fongicide de l'huile extraite de Ocimum gratissimum dénommée NECO, a été évaluée dans le traitement de la MRN en vue de développer contre cette maladie une option de lutte efficace pour un faible coût sans risque de contamination pour l'utilisateur, l'environnement et la production. 


\section{Kassi et al. J. Appl. Biosci. 2014. Action du fongicide naturel NECO contre la Mycosphaerella fijiensis Morelet chez le bananier plantain (AAB) en Côte d'Ivoire}

\section{MATERIEL ET METHODES}

Matériel et milieu d'étude : L'étude a été conduite en condition d'infestation naturelle de Mycosphaerella fijiensis et d'autres espèces de Mycosphaerella spp. entre Août 2010 et Avril 2011 sur la parcelle expérimentale du Laboratoire de physiologie végétal, propriété de l'Université Felix Houphouet Boigny. Les cultivars Orishele et Corne 1 localement très répandus et appréciés pour les caractéristiques organoleptiques de leurs fruits, ont été plantés. Ils sont respectivement très sensibles et sensibles à la MRN. Le site expérimental expérimental se situe à $5^{\circ} 58$ de latitude nord et $3^{\circ} 59$ de longitude ouest, avec une température moyenne de $26,8^{\circ} \mathrm{C}$, une humidité relative de $84 \%$, et $900,4 \mathrm{~mm}$ de pluie tombées au cours de la période de l'essai.

Dispositif expérimental et gestion agronomique : Un dispositif en blocs de Fisher a été utilisé avec 2 traitements, 3 répétitions et 6 parcelles comportant 20 plants chacune. La plantation a été faite le 02 Août 2010 avec des rejets d'environ $400 \mathrm{~g}$, en laissant un espace de $2 \mathrm{~m}$ aussi bien entre les lignes qu'entre les plants. Chaque parcelle était composée de deux lignes de 5 bananiers de chacun des cultivars (Corne 1 et Orishele). Les parcelles utiles étaient bordées par des plants du cultivar Corne 1. Au cours de l'expérimentation afin d'amplifier la maladie dans l'environnement aucun effeuillage n'a été effectué tout au long du cycle (Gauhl, 1994). En effet, les feuilles sèches qui restent collées à la plante représentent un excellent foyer d'inoculum (Gauhl, 1994). Pour le contrôle des mauvaises herbes, le fauchage tous les 3 mois à partir de la plantation a été la méthode mécanique adoptée et un apport de $50 \mathrm{~g}$ d'urée/ plant au $5^{\mathrm{e}}$ mois a été réalisé. La durée de l'expérience, de la plantation à la fin des évaluations fut de 9 mois.

Application du biofongicide (NECO) : Les 2 traitements évalués étaient d'une part le NECO et d'autre part le témoin (sans application). Le fongicide d'origine naturel NECO a été obtenu à partir des feuilles de Ocimum gratissimum récoltées frais. Ces feuilles ont subi une extraction par entrainement à la vapeur d'eau réalisée avec le dispositif de Clevenger (Oussou, 2009) pendant 2 heures pour produire l'huile essentielle. Le NECO a été pulvérisé à $5 \mathrm{ml} / \mathrm{L}$ après dissolution dans de l'huile minérale Banole qui sert de solvant. Le mélange NECO + Banole a été appliqué au feuillage tôt le matin à l'aide d'un pulvérisateur réglé à $1,072 \mathrm{ml} /$ pression. Au cours de l'évaluation de l'effet du NECO sur la vitesse d'évolution de la maladie des raies noires, 4 traitements ont été appliqués aux dates suivantes : traitement $1(23 / 11 / 10)$, traitement 2 $(07 / 12 / 10)$; traitement $3(08 / 01 / 11)$ et traitement 4 (02/03/11).

Évaluation de la maladie des raies noires en champ Des évaluations hebdomadaires de tous les traitements ont été pratiquées sur tous les plants de chaque répétition à partir du troisième mois (la 11ème semaine) qui a suivi la plantation. Les descripteurs phytopathologiques suivants ont été évalués :

- L'évaluation de l'état d'évolution de la maladie (EEM) a permis de quantifier le développement de la MRN sur l'essai. Le calcul de l'EEM ou de la vitesse d'évolution de la maladie en fonction du temps est caractérisé par un coefficient de base qui traduit le couple (rang de feuille - stade cercospora). Cette vitesse d'évolution de la maladie en relation avec les conditions climatiques et l'intensité de l'infection a été suivie et a permis de décider de l'application du biofongicide NECO lorsque la valeur de l'EEM a atteint 2000 unités. Suivant la méthode de calcul de l'EEM de Fouré et Ganry (2008) ,10 bananiers ont été choisis et observés sur l'ensemble des parcelles traités avec le NECO. Chaque semaine, les premières feuilles du haut vers le bas ont été évaluées sur chacun des bananiers choisis. Les observations ont consisté à noter : les stades de déroulement du cigare $(00,02,04,06,08)$ et le stade le plus évolué de la maladie sur les feuilles de rang 2 à 4 (FII, FIII, FIV). L'état d'évolution de la maladie (EEM) est alors déterminé par la formule suivante :

$$
\text { EEM }=\text { REF } \times(S B-C E)
$$

\section{Où}

REF = rythme d'émission foliaire exprimant la vitesse de croissance du bananier en fonction du temps ; $S B=$ somme brute caractérisant l'évolution de la maladie sans tenir compte du rythme d'émission foliaire; $\mathrm{CE}=$ coefficient d'évolution obtenu en multipliant la valeur du stade cigare par le nombre de feuilles présentant les différents stades de la maladie ; avec SB - CE correspondant à la somme corrigée notée SEV (Fouré \& Ganry, 2008).

- L'indice de sévérité (IS) de la maladie déterminé chaque 30 jours à partir du troisième mois après la plantation à l'aide de la méthode de Stover modifiée par Gauhl et al. (1995), a été calculé selon la formule suivante :

Indice de Sévérité $=\frac{\sum n b}{(N-1) T} \times 100$ 


\section{Kassi et al. J. Appl. Biosci. 2014. Action du fongicide naturel NECO contre la Mycosphaerella fijiensis Morelet chez le bananier plantain (AAB) en Côte d'Ivoire}

Où

$\mathrm{n}=$ nombre de feuilles de chaque degré ;

$b=$ degré $(0=$ pas de symptôme ;

$1=$ moins de $1 \%$ du limbe présentant des symptômes nécrotiques ;

$2=1$ à $5 \%$ du limbe avec des symptômes ;

$3=6$ à $15 \%$ du limbe avec des symptômes ;

$4=16$ à $33 \%$ du limbe avec des symptômes ;

$5=34$ à $50 \%$ du limbe avec des symptômes

$6=51$ à $100 \%$ du limbe avec des symptômes) ;

$\mathrm{N}=$ nombre de degrés employé dans l'échelle (7)

$T=$ nombre total de feuilles évaluées.

- La plus jeune feuille touchée (PJFT), c'est à dire en comptant les feuilles de haut en bas, la plus jeune

\section{RESULTATS}

Dans les parcelles témoins de Orishele et Corne 1, l'état d'évolution de la maladie (EEM) est progressif pendant toute la période d'observation (Figure 1). Les aspersions du fongicide d'origine naturel NECO dans les parcelles traitées ont entrainé la diminution de l'état d'évolution de la maladie des raies noires par rapport aux parcelles témoins n'ayant pas reçu de traitement. L'EEM se caractérise par des fluctuations d'une semaine à l'autre qui ont des amplitudes plus importantes chez le cultivar Orishele. Les valeurs de l'EEM chez Orishele sont moins importantes de la $22^{\mathrm{e}}$ à la 26 e semaine mais demeurent supérieure à celles du feuille portant les premiers symptômes de la maladie (au moins 10 tirets de stade I ; Fouré, 1983) ;

- la plus jeune feuille portant le stade III de la maladie (PJF3), mentionnée par Meredith et Lawrence, 1970 ;

- la plus jeune feuille nécrosée (PJFN), qui correspond rang de la plus jeune feuille présentant au moins 10 nécroses de stade $\mathrm{V}$ ou $\mathrm{VI}$ pour la cercosporiose noire (Bureau, 1984)

Analyses statistiques : Les données ont été analysées statistiquement grâce au logiciel Statistica 7.1 et au seuil de comparaisons des moyennes de Newman keuls avec 5 p.c. de probabilité.

cultivar Corne 1. La Figure 1 montre que les applications de NECO chez les deux cultivars maintiennent l'état d'évolution de la maladie des raies noires en dessous de 2000 unités (valeur seuil) avec une chute importante du niveau d'évolution de la maladie à chaque application. Suite à l'application du NECO à la 20 semaine, la valeur de l'EEM diminue de 2500 à 1000 unités à la $25 \mathrm{e}$ semaine pour le cultivar Corne 1. Durant cette même période dans les parcelles témoins la valeur de l'EEM est passée de 3000 et 2000 unités.

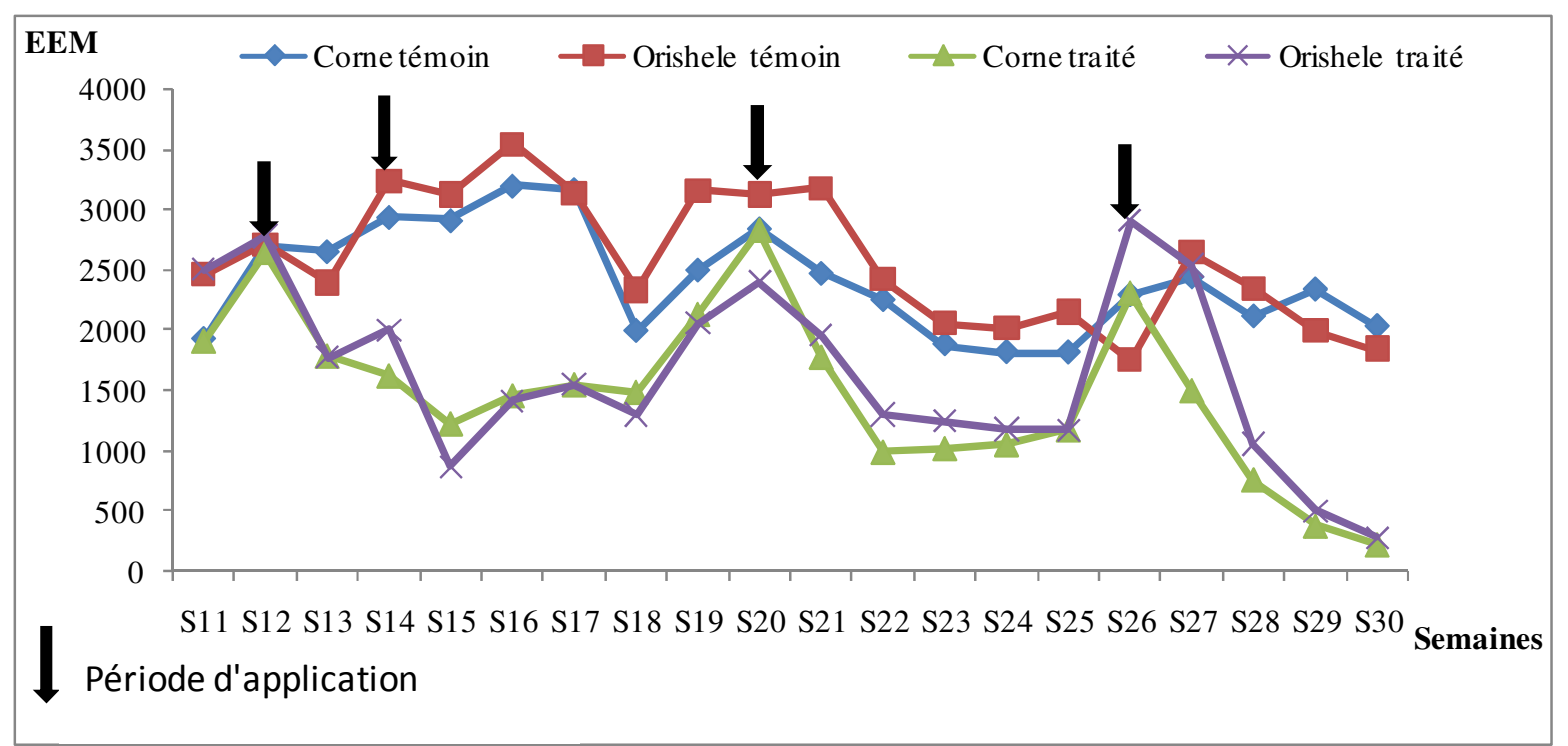

Figure 1 : Évolution Évolution hebdomadaire de l'état de la maladie des raies noires (MRN) chez différents cultivars de bananiers avec ou sans application de NECO 


\section{Kassi et al. J. Appl. Biosci. 2014. Action du fongicide naturel NECO contre la Mycosphaerella fijiensis Morelet chez le bananier plantain (AAB) en Côte d'Ivoire}

Des différences statistiques hautement significatives ont été observées pour les paramètres évaluées entre les traitements dans cette étude (Tableau 1). Le traitement à base du NECO a révélé les valeurs les plus élevées des plus jeunes feuilles touchée (PJFT), nécrosée (PJFN) de stade 3 (PJF3) mais les valeurs les plus faibles d'indice de sévérité (IS) contrairement au témoin aussi bien chez Orichele et Corne 1. Le rang de la plus jeune feuille touchée est situé entre 2 et $5 ; 2$ et 6 respectivement pour les cultivars Orishele et Corne 1 dans les parcelles traitées (Figure 2). Pour le rang de la plus jeune feuille présentant le stade 3 de la maladie, des différences prononcées ont également été obtenues entre les parcelles traitées et les parcelles témoins (Figure 3). II varie entre les feuilles de rang5 à 10 et 4 à 6 respectivement dans les parcelles traitées et les parcelles témoins.

Tableau 1 : Moyennes comparées des PJFT, PJF3, PJFN et de l'IS de la maladie des raies noires au cours de la période d'évaluation.

\begin{tabular}{lllcc}
\hline Traitements & PJFT & PJF3 & PJFN & IS \\
\hline Corne témoin & $2,13 \mathrm{c}$ & $4,79 \mathrm{c}$ & $6,23 \mathrm{cb}$ & $22,11 \mathrm{a}$ \\
Orishele témoin & $2,16 \mathrm{c}$ & $4,43 \mathrm{~d}$ & $5,96 \mathrm{c}$ & $23,33 \mathrm{a}$ \\
Corne traité & $2,89 \mathrm{a}$ & $6,64 \mathrm{a}$ & $8,15 \mathrm{a}$ & $3,26 \mathrm{~b}$ \\
Orishele traité & $2,65 \mathrm{~b}$ & $5,51 \mathrm{~b}$ & $6,47 \mathrm{~b}$ & $3,91 \mathrm{~b}$ \\
\hline
\end{tabular}

Dans chaque colonne, des moyennes affectées de lettres différentes montrent des différences statistiquement significatives d'après le test de comparaison de Newman-keuls au seuil de $5 \%$

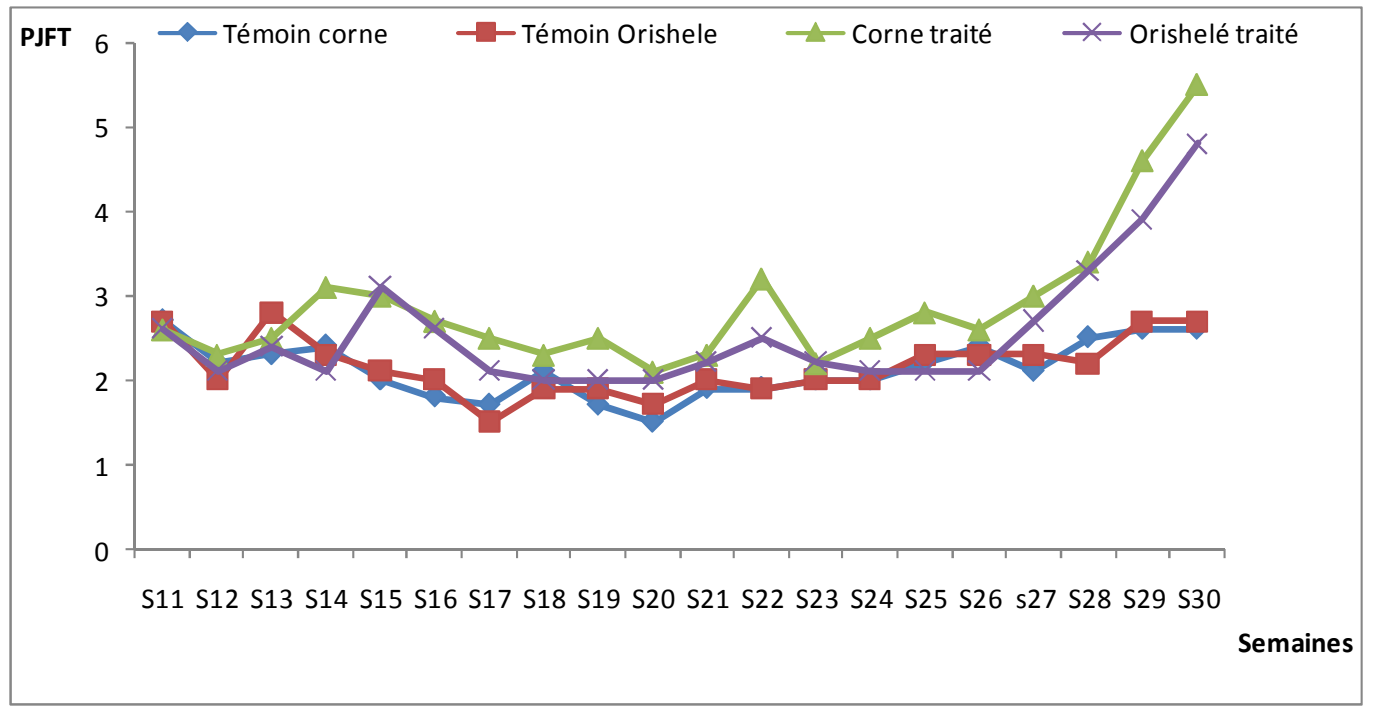

Figure 2 : Évolution hebdomadaire du rang de la plus jeune feuille touchée (PJFT) chez différents cultivars de bananiers avec ou sans application de NECO 


\section{Kassi et al. J. Appl. Biosci. 2014. Action du fongicide naturel NECO contre la Mycosphaerella fijiensis Morelet chez le bananier plantain (AAB) en Côte d'Ivoire}

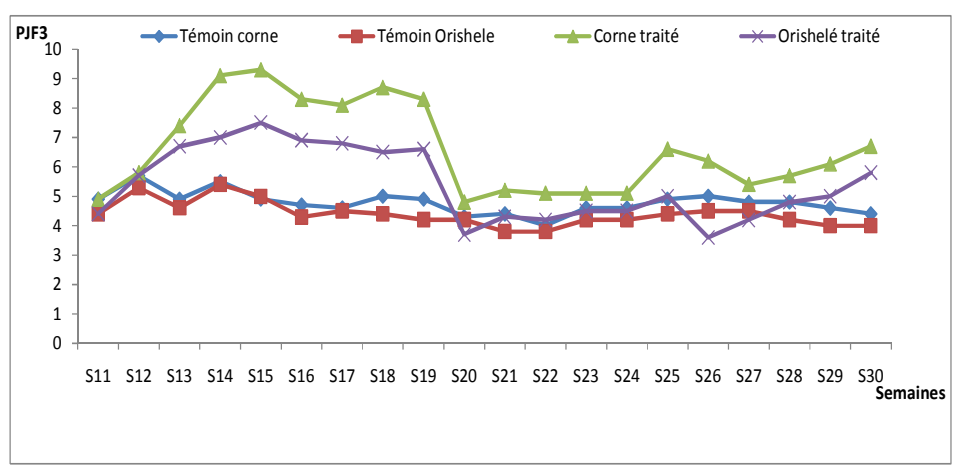

Figure 3 : Évolution hebdomadaire du rang de la plus jeune feuille portant le stade 3 (PJF3) de la MRN chez différents cultivars de bananiers avec ou sans application de NECO.

Des différences statistiques hautement significatives sont observées entre les parcelles traitées et les parcelles témoins pour la variable de la plus jeune feuille nécrosée. La PJFN est comprise entre les feuilles de rang 6 à 8 dans les parcelles témoins. Dans les parcelles traitées, la PJFN est de rang supérieur à 6 notamment chez le cultivar Corne 1 où elle a atteint la valeur 10 à la 20 ème semaine après le planting. Sur les parcelles témoins, le rang de la PJFN était généralement plus faible que sur les parcelles traitées par le NECO (Figure 4). L'indice de sévérité est plus élevé dans les parcelles témoins avec des valeurs allant de 19 à $30 \%$ (Figure 5). Dans les parcelles traitées par le NECO, la variation de l'indice d'infection a été très faible durant la période d'observation, il est resté inférieur à $8 \%$ et s'est annulé à la $16^{\circ}$ semaine.

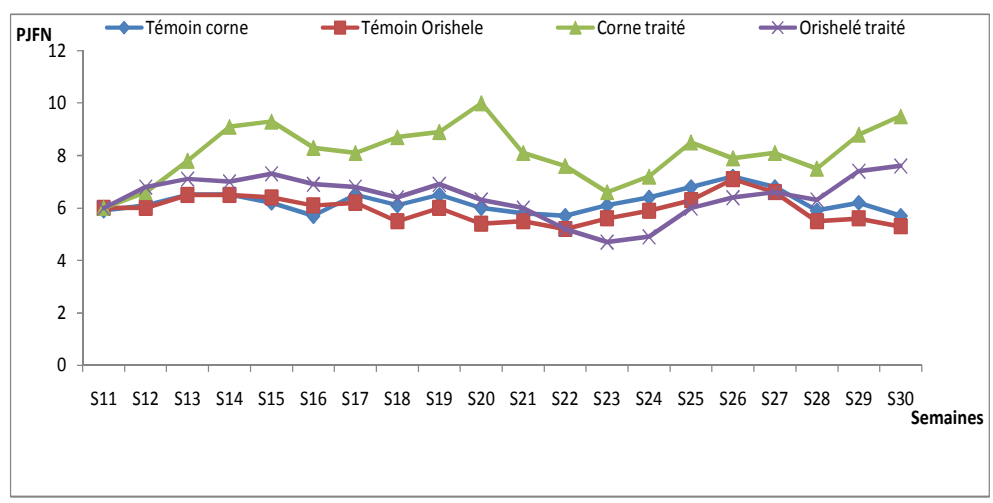

Figure 4 : Évolution hebdomadaire du rang de la plus jeune feuille nécrosée (PJFN) chez différents cultivars de bananiers avec ou sans application de NECO 


\section{Kassi et al. J. Appl. Biosci. 2014. Action du fongicide naturel NECO contre la Mycosphaerella fijiensis Morelet chez le bananier plantain (AAB) en Côte d'Ivoire}

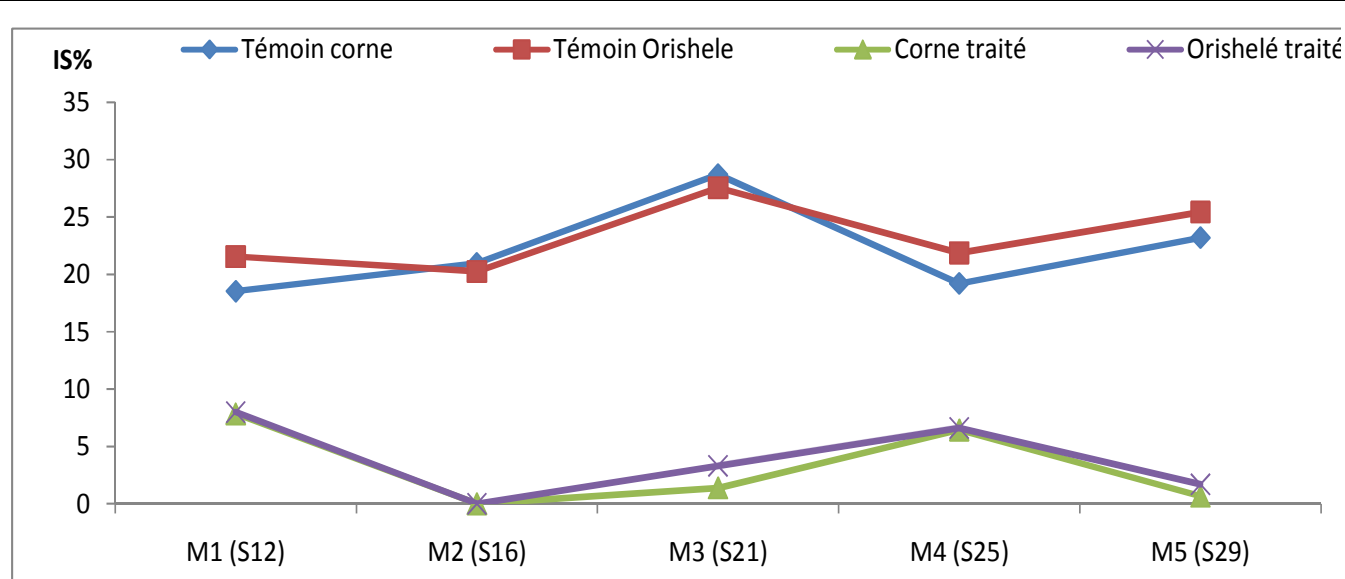

Figure 5 : Évolution mensuelle de l'indice de sévérité (IS) de la maladie des raies noires chez différents cultivars de bananiers avec ou sans application de NECO

\section{DISCUSSION}

L'évaluation de la réponse des bananiers aussi bien du cultivar Corne 1 que de Orishele au traitement par le NECO pour tous les paramètres caractérisant les symptômes foliaires de la MRN (EEM, PJFT, PJF3, PJFN et IS) a révélé des niveaux d'infection par la cercosporiose noire plus faibles dans les parcelles traitées que témoins. Les niveaux d'évolution de la maladie les plus élevés $\left(12^{\mathrm{e}}, 16^{\mathrm{e}}\right.$ et $19^{\mathrm{e}}$ semaines après la plantation) ont coïncidé avec les valeurs d'humidités relatives moyennes supérieures à $85 \%$ observées pour des niveaux de précipitations cumulées les plus élevés. Ces observations concordent avec celles faites par Aguirre et al. (1998a) mettant en évidence une corrélation positive entre la précipitation et la sporulation des agents pathogènes qui cause les symptômes de la maladie. Les bananiers des parcelles traitées par le biofongicide NECO se sont révélés moins sujets à l'infection de Mycosphaerella fijiensis, agent responsable de la cercosporiose noire que ceux des parcelles témoins. Les premiers symptômes (rang de la PJFT) ont été majoritairement observés sur les feuilles de rang 2 dans les parcelles témoins et de rang 3 dans les parcelles traitées, rendant ainsi compte de la baisse de la pression parasitaire suite aux applications du NECO. Pour ce paramètres (PJFT) les bananiers traités avec le NECO, ont eu une feuille saine supplémentaire par rapport aux plants non traités ce qui montre que le biofongicide NECO retarde l'évolution des symptômes de la maladie. Cependant, une phytotoxicité a été observée sur les feuilles (décoloration orange) dans les parcelles traitées par le
NECO qui était appliqué avec l'huile minérale le Banole. Avec l'activateur de défense, l'Acibenzolar associé à une huile vaporisée des auteurs ont aussi observé une phytotoxicité sur les plus vieilles feuilles des plants de bananiers pour des dosages de 3,6 L/ha (Vawdrey \& Grice, 2005) ou supérieurs à 5 L/ha (Madrigal, 1988), pouvant altérer les feuilles. La plus grande tolérance à la MRN avec ou sans traitement par le NECO du cultivar Corne 1 par rapport rapport au cultivar Orishele, s'expliquerait par sa densité stomatique moins importante (Vasquez et al., 1989). Cette tolérance est davantage renforcée avec l'application du fongicide naturelle NECO. En effet, les aspersions de NECO dans les parcelles traitées coïncident avec une chute de la vitesse d'évolution de la maladie et le maintient de l'indice d'infection à un bas niveau. Cet effet suggère ainsi une participation active des molécules du NECO dans l'inhibition directe du pathogène. En effet, les composés phénoliques tels que le thymol ; majoritairement présent dans l'huile de Ocimum gratissimum sont reconnus toxiques et auraient pour cible les enveloppes des microorganismes telles que la membrane cytoplasmique et la paroi (Uribe et al., 1985). C'est également le cas du 1,8- cinéole et du camphre qui inhibent la germination des organes de propagation ou d'infection et la croissance des agents pathogènes issus de ces organes (Ishikawa et al., 1986). Les travaux de Hamilton-kemp et al., 2000 ont également montré que les constituants de l'huile essentielle tels que les composés phénoliques, l'alcool terpénique et les 


\section{Kassi et al. J. Appl. Biosci. 2014. Action du fongicide naturel NECO contre la Mycosphaerella fijiensis Morelet chez le bananier plantain (AAB) en Côte d'Ivoire}

aldéhyde altèrent la perméabilité des membranes, dénaturent et précipitent les protéines. Les travaux de Harshini et al., (2008) ont montré la capacité des huiles essentielles de Ocimum basilicum et Cymbopogon citratus à inhiber la croissance des appressoria de Fusarium proliferatum et Colletotrichum musea, responsables de la pourriture de la couronne des bananes. La baisse continue de la vitesse d'évolution de la maladie des raies noires audelà de 4 semaines et malgré une humidité favorable à la réduction de la période d'incubation du champignon (13 à 14 jours) suggèrerait que le NECO pulvérisé sur les plants des parcelles traitées aurait en plus de son effet direct un effet indirect qui s'estompe après 5 semaines. Généralement, la relation entre une accumulation de composés conjugués et la résistance induite chez certaines plantes a déjà été bien établie

\section{REMERCIEMENTS}

Nous remercions sincèrement le Professeur Dick Emmanuel (Laboratoire de Physiologie Végétale, Université Félix Houphouet Boigny, Abidjan) pour son

\section{REFRENCES}

Adjanohoun EJ, Ake AL, 1979. Contribution au recensement des plantes médicinales de Côte d'Ivoire

Aguirre MC, Castano- zapata J, Valencia JA, Zuluaga LE, Arce C, 1988 a. Interaccion de Mycosphaerella fijiensis Morelet y M. musicola Leach en siete genotipos de la Sigatoka negra en la zonacafetera colombiana. Pp. 192-220 in Memorias del Seminaio Internacional sobre $P$ roduction de Platano. Universidad del QuindioSENA-INABAP-CORPOICA

Amari LDGE, 2012. Stratégies d'évaluation et de gestion par stimulation des défenses naturelles des bananiers à l'infection de la maladie des raies noires causée par Mycosphaerella fijiensis Morelet (Mycosphaerellacées) en Côte d'Ivoire. Thèse Unique de Doctorat en Physiologie Végétale, Université Félix HOUPHOUÊT-BOIGNY. 237 p.

Beveraggi A, Mourichon X and Sallé G, 1995. Etude compare des premières étapes de l'infection chez des bananiers sensibles et résistantes infectés par Cercospora fijiensis, agent de la maladie des raies noires. Can. J. Bot., 73, 1328- 1337. dans d'autres pathosystèmes et est évoquée pour être une composante nécessaire à la réponse défensive de l'hôte (Ongena et al., 2000 ; Amari, 2012). L'effet éliciteur des extraits de feuilles de Ocimum gratissimum a été mis en évidence chez le sorgho et le soja respectivement par une induction de la production de deoxyanthocyanidines et de phytoalexines (Colpas et al., 2009). L'implication des proanthocyanidines dans la résistance partielle de certains bananiers a été révélée dans les travaux de Beverraggi et al. 1995 ; El Hadrami 1997. Les résultats de ce travail indiquent que l'utilisation du NECO se présente donc comme une option efficace et viable dans la lutte contre la maladie des raies noires causées par Mycosphaerella fijiensis avec pour avantage d'être économique et non toxique pour l'agriculteur, le consommateur et l'environnement.

apport personnel et financier pendant nos travaux. Notre gratitude va aussi à l'endroit des responsables du Laboratoire de Physiologie Végétale.

Bureau E, 1984. L'avertissement climatique dans le cadre d'une stratégie nouvelle de traitement contre la cercosporiose du bananier en Gouadeloupe. Fruits vol. $37, n^{\circ} 7-8 ;$ p. 441447

Camara B, 2011. Caractérisation des parasites fongiques foliaires et telluriques en Côte d'lvoire chez les bananiers (Musa sp.) et recherche de méthodes de lutte. Thèse de Doctorat Unique. Laboratoire de Physiologie Végétale, UFR biosciences, Université de Cocody-Abidjan. $219 p$

Camara B, Koné D, Kanko C, Anno A, Ake S, 2007. Activité antifongique des huiles essentielles de Ocimum gratissimum L., de Monodora myristica (Gaaertn) Dunal et de deux produits de synthèses (Impulse et Folicur), sur la croissance mycélienne et la production de spore in vitro de Deightoniella torulasa (SYD.) ELLIS

Chillet M, Abadie C, Hubert O, Chilin-Charles Y, de Lapeyre de Bellaire L, 2009. Sigatoka disease reduces the greenlife of bananas. Crop Protection, 28 (1) : 41-45

Clevenger J, 1928. Apparatus for volatile oil determination, Description of New Type. 


\section{Kassi et al. J. Appl. Biosci. 2014. Action du fongicide naturel NECO contre la Mycosphaerella fijiensis Morelet chez le bananier plantain (AAB) en Côte d'Ivoire}

American Perfumer \& Essential Oil Review, 1928, 467-503.

Colpas FT, Schwan-estrada KRF, Sangarlin JR, Ferrarese ML, Scapim CA, Bonaldo SM, 2009. Induction of plant defense responses by Ocimum gratissimum L. (Lamiaceae) leaf extracts. Summa phytopathologica, v.35,n.3, pp. 191-195

El Hadrami A, 1997. Proanthocyanidines constitutives des feuilles de bananiers et résistance partielle vis-à-vis de Mycosphaerella fijiensis, l'agent causal de la maladie des raies noires. Mémoire : Faculté universitaire des Sciences agronomiques de Gembloux (Belgique)

Essis B, Kobenan K, Traore S, Koné D and Yatty J, 2010. Sensibilité au laboratoire de Mycosphaerella fijiensis responsable de la cercosporiose noire des bananiers vis-à-vis de fongicides couramment utilisés dans les bananeraies ivoiriennes

Fouré E, 1983. Les cercosporioses du bananier et leurs traitements. Sélection de molécules fongicides nouvelles. Fruits, 38 (1) : 21-34

Fouré E, and Ganry J, 2008. A bilogical forecasting system to control Black Leaf Streak Disease of bananas and plantains. Fruits 63 (5) 311-317

Fullerton RA and Olsen TL, 1991. Pathogenic in Mycosphaerella fijiensis Morelet. In : Banana diseases in Asia and the Pacific (INHIBAP, FRA).

Gauhl F, 1994. Epidemiology and ecology of black Sigatoka (Mycosphaerella fijiensis Morelet) on plantain and banana in Costa Rica. INIBAP, Montpellier, France. $120 \mathrm{pp}$.

Hamilton-kemp, Archbold, 2000. Stimulation and inhibition of fungal pathogens of plants by natural valatile phytochemicals and their analogs. Current topics in phytochemistry, 4 , 95-104

Harshini Herath and Krishanti Abeywickrama, 2008. In vitro application of selected essential oils and their major components in controlling fungal pathogens of crown rot in Embul banana ( Musa acuminate- AAB

Ishikawa M, Shuto Y, Watanabe H, 1986. Agric. Biol. Chem., 50, pp. 1863, 1986

Kishore Dubey N, Tiwari TN, Mandin D, Andriamboavonjy $\mathrm{H}$ and Chaumont J-P, 2000. Antifungal properties of Ocimum gratissimum essential oil ( ethyl cinnamate chemotype). Fitoterapia, 71: 567-569.
Meredith DS, and Lawrence JS, 1970. Black leaf steak disease of banana (Mycosphaerella fijiensis) susceptibility of cultivars

Mouliom-Pefoura A, 1999. First observation of breakdown of resistance in Yamgambi $\mathrm{Km} 5$ (Musa sp.) to the black leaf streak disease in Cameroun. Plant Disease, 83: 78.

Mourichon X, Lepoivre P, and Carlier J, 2000. Black leaf streak. Host-pathogen interactions. Pp. 67-72 in Diseases of Banana, Abacá and Enset. (D.R. Jones, ed.). CABI Publishing,Wallingford, UK

Mourichon X, and Fullerton R, 1990. Geographical distribution of two species Mycosphaerella musicola Leach (Cercospora musae) and $M$. fijiensis morelet (Cercospora fijiensis), respectively agents of Sigatoka and Black leaf streak diseases in bananas and plantains. Fruits 45: 213-218

Ongena $M$, Daayf $F$, Jacques $P$, Thonart $P$, Benhamou $\mathrm{N}$, Paulitz TC, and Belanger RR, 2000. Systemic induction of phytoalexins in cucumber in response to treatments with fluorescent Pseudomonads. Plant Pathol. 49(4):523-530

Oussou KR, 2009. Étude chimique et activités biologiques des huiles essentielles de sept plantes aromatiques de la pharmacopée ivoirienne. Thèse de Doctorat Unique. Laboratoire de chimie organique et biologique, UFR SSMT, Université de Cocody-Abidjan. $241 p$

Oussou KR, Kanko C, Guessennd N, Yolou S, Koukoua G, Dosso M, N'Guessan YT, Figueredo G, Chalchat JC, 2004. Activités antibactériennes des huiles essentielles de trois plantes aromatiques de Côte d'lvoire ; C.R. Chimie pp 292-297

Pasberg-gauhl C, and Gauhl F, 2000.Response to east African Highland Bananas to black leaf streak sigatoka and Cladosporium leaf speckle under tropical humid forest and conditions in West Africa. In: Banana and Plantain for Africa. K. Craenen et al.(Eds). Proc. I. Int. Symp Acta Hort. $540 \mathrm{p}$.

Ramsey MD, Daniells JW, Anderson VJ, 1990. Effects of sigatoka leaf spot (Mycospherella musicola Leach) on fruit yield, field ripening and greenlife of bananas in North Queensland. Scientia Horticulturae 41: 305-313 
Rodriguez R, and Jimenez L, 1985. El problema de la tolerancia de Mycosphaerella fijiensis fungicida benomil en plantaciones bananeras de Costa Rica. ASBANA. 16 pp.

Sanchez Rodriguez R, Pino Algora JA, Vallin Plous C, Pérez Rodruez ME, Iznaga Sosa and Malpartida Romero F, 2002. Action du fongicide naturel F20 contre la cercosporiose noire (Mycosphaerella fijiensis Morelet) chez le bananier plantain (AAB) et la bananier (AAA). INFOMUSA 11 (1) : 14-16.

Stover RH, 1980. Sigatoka leaf spots of bananas and plantains. Plant Disease 64: 750-755.

Uribe S, Ramirez J, and Pena A, 1985. Effet of a-pinen on Yeast membrane functions. Journal of Bacteriology vol. 161, $n^{\circ} 3$ pp. 1195-11200.

Vasquez N, Tapia AC, and Galindo JJ, 1989. Ultrastructural studies of the infection of Mycosphaerella on Musa cultuvar. In: " Fullerton (R.A.) and (R.H.) Stover. 1989. Sigatoka leaf spot diseases of banana ». Proceedings of an international workshop at an José, Costa Rica, March 28- April

Vawdrey LL, and Grice K, 2005. Evaluation en champ de l'action des strobilurines, des triazoles et de l'acibenzolaar pour lutter contre la maladie de Sigatoka en Australie. InfoMusa 14 (2) 11-15. 